\title{
INCIDÊNCIA DE COLELITÍASE EM NECROPSIAS REALIZADAS EM HOSPITAL UNIVERSITÁRIO NO MUNICÍPIO DE CAMPINAS-SP
}

\author{
INCIDENCE OF CHOLELITHIASIS: A NECROPSY STUDY \\ PERFORMED IN CAMPINAS UNIVERSITY HOSPITAL, SP
}

\author{
Mario Mantovani, TCBC-SP 1 \\ Raquel Franco Leal ${ }^{2}$ \\ Mauro José Fontelles ${ }^{3}$
}

\begin{abstract}
RESUMO: Objetivo: avaliar a incidência de colelitíase em pacientes submetidos à necropsia no Hospital das Clínicas da UNICAMP e relacioná-la com a ocorrência de outras doenças associadas. Método: Os autores analisaram a incidência de colelitíase em 2.355 necropsias realizadas pelo Departamento de Anatomia Patológica da UNICAMP, no período de 1975 a 1998, considerando-se somente os casos com idade acima de 10 anos. O teste do qui-quadrado e a "odds ratio" (OR) foram utilizados para análise de correlação com outras afecções. Resultados: A incidência foi de $243(10,3 \%)$ casos de colelitíase; com $110(7,9 \%)$ casos em homens e $133(13,6 \%)$ em mulheres $(\mathrm{p}=0,00001)$, resultando numa proporção de 1:1,7. A incidência aumentou com a idade $(\mathrm{p}<0,000000001)$ e diferiu, significativamente, entre os grupos raciais estudados, sendo $195(11,1 \%)$ casos em indivíduos da raça branca e $48(7,8 \%)$ em não brancos $(\mathrm{p}=0,02)$. Quando relacionada com as demais doenças hepáticas associadas, observou-se que as de maior incidência foram a esteatose, com 33 (13,5\%) casos; as neoplasias, com 31 (12,7\%); a cirrose, com 30 (12,3\%); a hepatite e a congestão crônica do fígado, cada uma com 16 (6,5\%) casos, respectivamente. $\mathrm{Na}$ análise de correlação verificou-se que as neoplasias, o infarto hepático e a atrofia parda do fígado mostraram associações estatisticamente significantes com a incidência de colelitíase. Conclusões: Os resultados indicam um aumento progressivo na incidência de colelitíase com a idade, sendo mais incidente na população acima dos 80 anos e, com predomínio, em indivíduos da raça branca.
\end{abstract}

Descritores: Colelitíase; Vesícula biliar; Necropsia.

\section{INTRODUÇÃO}

A freqüência da calculose biliar, nos diversos países em que foi estudada, é muito variável ${ }^{1-5}$. No Brasil, apesar de sua dimensão continental, até 1998 , foram realizadas apenas duas pesquisas referentes à incidência de colelitíase em material de necropsia, feitas por Rocha ${ }^{5}$, no Triângulo Mineiro, e por Nakaie ${ }^{6}$, em São Paulo.

$\mathrm{Na}$ literatura especializada, com inúmeros trabalhos publicados sobre o assunto, a epidemiologia da colelitíase assume papel de destaque, podendo levar à chave da etiologia desta importante afecção ${ }^{6-8}$. Assim, entre os possíveis métodos de avaliação, o estudo clínico é de uso limitado para a determinação da prevalência da calculose biliar, pois cerca de $60 \%$ a $80 \%$ dos pacientes permanecem assintomáticos e, portanto, não diagnosticados ${ }^{4-6}$. Os estudos por imagem, sejam por ultra-sonografia ou radiológicos, embora casualizados, são procedimentos onerosos para ser empregados em larga escala, sendo o material de necropsia, ainda que apresente limitações, o mais freqüentemente

1. Professor Titular e Chefe da Disciplina de Cirurgia do Trauma do Departamento de Cirurgia da Faculdade de Ciências Médicas da UNICAMP.

2. Acadêmica da Faculdade de Ciências Médicas da UNICAMP.

3. Mestre em Cirurgia do Trauma e Aluno do Curso de Doutorado do Departamento de Cirurgia da Faculdade de Ciências Médicas da UNICAMP.

Recebido em 8/8/2000

Aceito para publicação em 6/3/2001

Trabalho realizado pela Disciplina de Cirurgia do Trauma do Departamento de Cirurgia da Faculdade de Ciências Médicas da UNICAMP - Campinas-SP 
utilizado $^{9}$, em especial, quando empregada uma amostra de pacientes hospitalizados e avaliados pelos serviços de verificação de óbitos, o que torna o método preciso e com resultados fidedignos.

Deste modo, o objetivo do presente estudo foi o de avaliar a incidência de colelitíase em pacientes submetidos à necropsia no Hospital das Clínicas da UNICAMP e relacioná-la com a ocorrência de outras doenças associadas.

\section{MÉTODO}

Analisaram-se 2.355 necropsias realizadas pelo Departamento de Anatomia Patológica da Faculdade de Ciências Médicas da Universidade Estadual de Campinas, no período de 1975 a 1998, em pacientes internados e falecidos no Hospital de Clínicas; através de consulta no livro de registro e relatório de autópsias, com análise de dados relativos ao sexo, idade, raça, doenças associadas e causa mortis. Não foram incluídos no estudo indivíduos menores de 10 anos de idade.

Com os resultados encontrados, um banco de dados foi desenvolvido, utilizando-se o programa EPI INFO versão 6.04b (Center for Disease Control \& Prevention, USA - 1997), onde todas as informações foram cadastradas, possibilitando, a qualquer tempo, a pesquisa e o cruzamento estatístico entre os dados coletados.

As análises de correlação foram realizadas através da aplicação do teste de qui-quadrado em tabelas de contingência de dupla entrada. Tratando-se com freqüências menores que cinco ou com pequenas amostragens, este teste foi substituído pelo teste de probabilidade exata de Fisher, a fim de se evitarem distorções na apreciação da significância estatística. A "odds ratio" foi utilizada para o cálculo das chances de associações da litíase biliar com as demais afecções hepáticas. Em todas as análises empregou-se o nível alfa de $5 \%(\mathrm{p} \leq 0,05)$.

\section{RESULTADOS}

Estudando as 2.355 necropsias realizadas, encontrou-se uma incidência de litíase biliar em 243 (10,3\%) casos. Entre os indivíduos estudados, $1.382(58,6 \%)$ pertenciam ao sexo masculino e $973(41,4 \%)$ ao sexo feminino. A incidência por sexo foi de $110(7,9 \%)$ casos em homens e $133(13,6 \%)$ em mulheres, resultando numa proporção de 1:1,7 (Tabela 1).

Em relação à faixa etária, verificou-se que a incidência de cálculos da vesícula biliar aumentou com a idade. Considerando-se os casos com idade acima de 20 anos, a incidência de litíase biliar aumentou para $9,0 \%$, no sexo masculino, e $15,7 \%$, no sexo feminino, com incidência de $11,7 \%$, no total dos casos (Tabela 2).

O material foi analisado tendo em conta o aspecto racial da população. Como há dificuldades em caracterizar o grupo mestiço e o reduzido número de indivíduos da raça amarela, considerou-se como relatados na literatura e, para efeito estatístico, apenas dois grupos: "brancos" e "não brancos". Desta forma, a proporção de indivíduos com calculose biliar diferiu, significativamente, entre os grupos
Tabela 1

Número de casos conforme o sexo e a incidência de litíase biliar em 2.355 necropsias

\begin{tabular}{|c|c|c|c|c|c|}
\hline \multirow[b]{3}{*}{ Sexo } & \multicolumn{4}{|c|}{ Litíase } & \multirow[b]{3}{*}{ Total } \\
\hline & \multicolumn{2}{|c|}{ Sim } & \multicolumn{2}{|c|}{ Não } & \\
\hline & $N^{o}$ & $\%$ & $N^{o}$ & $\%$ & \\
\hline Feminino & 133 & 13,6 & 840 & 86,4 & 973 \\
\hline Masculino & 110 & 7,9 & 1272 & 92,1 & 1382 \\
\hline Total & 243 & 2112 & 2355 & & \\
\hline$P=0,00001$ & & $=1,83$ & $I C$ & $(1,3$ & $-2,41)$ \\
\hline
\end{tabular}

Tabela 2

Número de casos conforme o grupo etário e a incidência de litíase biliar em 2.355 necropsias

\begin{tabular}{l|c|c|c|c|c}
\hline \multirow{2}{*}{$\begin{array}{l}\text { Grupo } \\
\text { etário } \\
\text { (anos) }\end{array}$} & \multicolumn{2}{|c|}{$\begin{array}{c}\text { Com } \\
\text { litíase }\end{array}$} & \multicolumn{2}{c|}{$\begin{array}{c}\text { Sem } \\
\text { litíase }\end{array}$} & \multirow{2}{*}{$\begin{array}{c}\text { Necropsias } \\
N^{o}\end{array}$} \\
\cline { 2 - 4 } & $N^{o}$ & $\%$ & $N^{o}$ & $\%$ & 298 \\
$10-19$ & 02 & 0,6 & 296 & 99,4 & 194 \\
$20-29$ & 07 & 3,6 & 187 & 96,4 & 297 \\
$30-39$ & 18 & 6,0 & 279 & 94,0 & 353 \\
$40-49$ & 28 & 7,9 & 325 & 92,1 & 420 \\
$50-59$ & 50 & 11,9 & 370 & 88,1 & 421 \\
$60-69$ & 62 & 14,7 & 359 & 85,3 & 271 \\
$70-79$ & 47 & 17,3 & 224 & 82,7 & 101 \\
$80-90$ & 29 & 28,7 & 72 & 71,3 & 2355 \\
\hline Total & 243 & 10,3 & 2112 & 89,7 & \\
\hline
\end{tabular}

$P<0,000000001$

étnicos estudados (com influência da raça sobre a incidência de colelitíase no nosso meio) (Tabela 3).

Quando relacionadas as demais doenças hepáticas associadas à colelitíase, observou-se que as de maior incidência foram: a esteatose com $33(13,5 \%)$ casos; as neoplasias, com 31 (12,7\%); a cirrose, com 30 (12,3\%); a hepatite e a congestão crônica do fígado, cada uma com 16 $(6,5 \%)$ casos, respectivamente. $\mathrm{Na}$ análise de correlação entre a colelitíase e estas afecções, verificou-se que, as neoplasias, o infarto hepático e a atrofia parda do fígado mostraram associações estatisticamente significantes. A "odds ratio" mostrou, na presente casuística, que a presença de calculose biliar nos indivíduos estudados aumentou em 1,55 vez a chance de evoluir com neoplasias hepáticas; em 7,05 vezes a chance de apresentarem infarto hepático e 17,52 vezes a chance da ocorrência de atrofia parda do fígado (Tabela 4). 
Tabela 3

Número de casos conforme os grupos raciais estudados e a incidência de litíase biliar em 2.355 necropsias

\begin{tabular}{|c|c|c|c|c|c|}
\hline \multirow{2}{*}{$\begin{array}{l}\text { Grupo } \\
\text { etário } \\
\text { (anos) }\end{array}$} & \multicolumn{2}{|c|}{$\begin{array}{l}\text { Com } \\
\text { litíase }\end{array}$} & \multicolumn{2}{|c|}{$\begin{array}{l}\text { Sem } \\
\text { litíase }\end{array}$} & \multirow{2}{*}{$\begin{array}{c}\text { Necropsias } \\
\quad N^{o}\end{array}$} \\
\hline & № & $\%$ & Noo & $\%$ & \\
\hline Brancos & 195 & 11,1 & 1550 & 88,9 & 1745 \\
\hline Não-brancos & 48 & 7,8 & 562 & 92,2 & 610 \\
\hline Total & 243 & 10,3 & 2112 & 89,7 & 2355 \\
\hline
\end{tabular}

Quanto às doenças associadas e não relacionadas ao sistema digestivo, as mais freqüentes, no total dos 243 casos de calculose da vesícula biliar, foram a aterosclerose, com $54(22,2 \%)$ casos; a nefrosclerose arteriolar benigna, com 43 (17,6\%); a broncopneumonia, com 31 (12,7\%); a insuficiência cardíaca congestiva, com $29(11,9 \%)$ e o enfisema pulmonar, com $22(9,0 \%)$ dos casos.

\section{DISCUSSÃO}

A incidência global de litíase biliar encontrada nesta casuística foi de $11,7 \%$, se considerados os casos acima de 20 anos de idade, sendo esta maior que no Triângulo Mineiro $^{5}(9,07 \%)$ e em Curitiba ${ }^{10}(9,3 \%)$, este último

Tabela 4

Número de casos conforme a incidência das doenças hepáticas e suas associações com a colelitíase em 2.355 necropsias

\begin{tabular}{|c|c|c|c|c|c|c|}
\hline \multirow[b]{2}{*}{ Doenças associadas } & & \multicolumn{2}{|c|}{ Litíase } & \multirow[b]{2}{*}{ Total } & \multirow[b]{2}{*}{$P$} & \multirow[b]{2}{*}{$O R$} \\
\hline & & Sim & Não & & & \\
\hline Esteatose & $\begin{array}{l}\text { Sim } \\
\text { Não }\end{array}$ & $\begin{array}{r}33 \\
210 \\
\end{array}$ & $\begin{array}{r}354 \\
1758 \\
\end{array}$ & $\begin{array}{r}387 \\
1968 \\
\end{array}$ & 0,23 & 0,78 \\
\hline Neoplasia & $\begin{array}{l}\text { Sim } \\
\text { Não }\end{array}$ & $\begin{array}{r}31 \\
212 \\
\end{array}$ & $\begin{array}{r}182 \\
1930 \\
\end{array}$ & $\begin{array}{r}213 \\
2142 \\
\end{array}$ & $0,04 *$ & 1,55 \\
\hline Cirrose & $\begin{array}{l}\text { Sim } \\
\text { Não }\end{array}$ & $\begin{array}{r}30 \\
213 \\
\end{array}$ & $\begin{array}{r}314 \\
1798 \\
\end{array}$ & $\begin{array}{r}344 \\
2011 \\
\end{array}$ & 0,33 & 0,81 \\
\hline Hepatite & $\begin{array}{l}\text { Sim } \\
\text { Não }\end{array}$ & $\begin{array}{r}16 \\
227\end{array}$ & $\begin{array}{r}138 \\
1974\end{array}$ & $\begin{array}{r}154 \\
2201\end{array}$ & 0,91 & 1,01 \\
\hline Congestão crônica & $\begin{array}{l}\text { Sim } \\
\text { Não }\end{array}$ & $\begin{array}{r}16 \\
227 \\
\end{array}$ & $\begin{array}{r}179 \\
1933 \\
\end{array}$ & $\begin{array}{r}195 \\
2160 \\
\end{array}$ & 0,37 & 0,76 \\
\hline Abscesso hepático & $\begin{array}{l}\text { Sim } \\
\text { Não }\end{array}$ & $\begin{array}{r}10 \\
233 \\
\end{array}$ & $\begin{array}{r}44 \\
2068 \\
\end{array}$ & $\begin{array}{r}54 \\
2301 \\
\end{array}$ & 0,07 & 2,01 \\
\hline Infarto hepático & $\begin{array}{l}\text { Sim } \\
\text { Não }\end{array}$ & $\begin{array}{r}04 \\
239\end{array}$ & $\begin{array}{r}05 \\
2107\end{array}$ & $\begin{array}{r}09 \\
2346\end{array}$ & $0,009 *$ & 7,05 \\
\hline Colestase & $\begin{array}{l}\text { Sim } \\
\text { Não }\end{array}$ & $\begin{array}{r}03 \\
240 \\
\end{array}$ & $\begin{array}{r}35 \\
2077 \\
\end{array}$ & $\begin{array}{r}38 \\
2317 \\
\end{array}$ & 0,79 & 0,74 \\
\hline Fibrose portal & $\begin{array}{l}\text { Sim } \\
\text { Não }\end{array}$ & $\begin{array}{r}03 \\
240\end{array}$ & $\begin{array}{r}26 \\
2086\end{array}$ & $\begin{array}{r}29 \\
2326\end{array}$ & 0,58 & 1,00 \\
\hline Atrofia parda & $\begin{array}{l}\text { Sim } \\
\text { Não }\end{array}$ & $\begin{array}{r}02 \\
241 \\
\end{array}$ & $\begin{array}{r}01 \\
2111 \\
\end{array}$ & $\begin{array}{r}03 \\
2352 \\
\end{array}$ & $0,02 *$ & $17, .52$ \\
\hline Hemangioma & $\begin{array}{l}\text { Sim } \\
\text { Não }\end{array}$ & $\begin{array}{r}01 \\
242\end{array}$ & $\begin{array}{r}15 \\
2097\end{array}$ & $\begin{array}{r}16 \\
2339 \\
\end{array}$ & 0,49 & 0,58 \\
\hline Cisto hepático & $\begin{array}{l}\text { Sim } \\
\text { Não }\end{array}$ & $\begin{array}{r}01 \\
242 \\
\end{array}$ & $\begin{array}{r}13 \\
2099 \\
\end{array}$ & $\begin{array}{r}14 \\
2341 \\
\end{array}$ & 0,56 & 0,67 \\
\hline Colangite & $\begin{array}{l}\text { Sim } \\
\text { Não }\end{array}$ & $\begin{array}{r}01 \\
242\end{array}$ & $\begin{array}{r}12 \\
2100\end{array}$ & $\begin{array}{r}13 \\
2342\end{array}$ & 0,60 & 0,72 \\
\hline
\end{tabular}

*Associações estatisticamente significantes

Constatou-se, ainda, que a maior incidência de hepatopatias associadas aos casos de litíase biliar ocorreu na faixa etária de 50 a 59 anos de idade, com 30 (12,7\%) casos e, na faixa etária de 60 a 69 anos, também, com 30 $(14,7 \%)$ casos. um estudo ultra-sonográfico, e menor que em São Paulo ${ }^{6}$ (19,3\%). Comparada aos estudos realizados em outros países, encontra-se próxima à da Itália ${ }^{11}(11,2 \%)$ e da Filadélfia $^{12}(11,6 \%)$ e, menor que a incidência encontrada na Suíça ${ }^{7}(17,6 \%)$. 
Os resultados obtidos confirmam que a incidência de colelitíase é maior no sexo feminino $(\mathrm{p}=0,0001)$, com proporção homem:mulher de 1:1,7, como é visto na literatura ${ }^{2,5,7,8,11,12,13}$, embora o estudo realizado em outra região do país ${ }^{5}$ tenha relatado uma proporção de 1:4. Estes resultados mostram que as mulheres, na presente casuística, apresentaram 1,83 vez mais chance de calculose biliar quando comparadas ao sexo masculino.

Nos diversos países, as publicações têm mostrado um aumento na incidência com a idade , $^{2,3,5,7,8,11-13}$. Neste trabalho, isto também foi verificado, pois houve aumento progressivo até a faixa etária acima de 80 anos ( $\mathrm{p}<0,000000001)$.

Considerando a importância do aspecto racial sobre a incidência da colelitíase, constatou-se que a proporção de indivíduos da raça branca $(11,1 \%)$ acometidos pela doença foi superior à encontrada nos indivíduos não-bran$\cos (7,8 \%)$, relação esta significativa $(p=0,02)$, demonstrando, assim, uma chance maior de evoluírem com calculose biliar, da ordem de 1,47 vez, se comparados aos demais grupos raciais. Estes resultados corroboram os valores encontrados nos $\mathrm{EUA}^{9,12}$

Neste trabalho, não foi possível a determinação da variável peso no material estudado; no entanto, a tendência de formação de cálculos, principalmente de colesterol, foi observada em pessoas obesas, em vários estudos da literatura ${ }^{4,14}$

A associação entre a litíase biliar e as demais doenças que acometem a população com faixa etária mais avançada é bastante referida nas publicações, porém, poucas destas relações são bem definidas após exame crítico mais acurado ${ }^{6}$. Observa-se, portanto, neste estudo, que as doenças não relacionadas ao sistema digestivo e que mais freqüentemente se associaram aos casos de colelitíase foram a aterosclerose, a nefroesclerose arteriolar benigna, a insuficiência cardíaca congestiva e o enfisema pulmonar que, igualmente à litíase biliar, também estão associadas às idades mais avan- çadas da população. Na literatura ${ }^{9,15}$, a aterosclerose é referida como doença relacionada à ocorrência de cálculo biliar, fato este não evidenciado no presente trabalho.

Bergman ${ }^{16}$ et al. observaram maior incidência de infarto do miocárdio em pacientes com colelitíase, no entanto, isto não foi verificado em nosso meio e no estudo em São Paulo, por Nakaie ${ }^{6}$ et al.

Dentre as doenças do aparelho digestivo, associadas à colelitíase, as mais frequientes foram as neoplasias do fígado, com $31(12,7 \%)$ casos, seguidas pela cirrose hepática, com 29 (11,9\%); e a pancreatite, com $24(9,8 \%)$ casos. Entre as patologias específicas do fígado, as mais freqüentes foram a esteatose, as neoplasias hepáticas e a cirrose.

Em relação à cirrose hepática, pela análise estatística, pode-se concluir que não houve associação com a litíase biliar. Isto também foi observado na Índia ${ }^{17}$, em 1989; no Triângulo Mineiro ${ }^{05}$ e em São Paulo ${ }^{4}$, no ano de 1982. Em estudos realizados na América do Norte ${ }^{12,18}$, na Europa $^{19,20,21}$ e no Brasil, por Genzini ${ }^{13}$, esta associação foi estatisticamente significante.

A associação entre litíase e câncer da vesícula biliar, é bem estabelecida, pois $65 \%$ a $100 \%$ dos casos de neoplasia vesicular têm cálculos. Nesta casuística, verificou-se que, em 2.355 autópsias, apenas sete $(0,29 \%)$ casos de câncer da vesícula biliar foram encontrados, representando uma incidência de 7,2\% entre todos os casos de neoplasia, nos indivíduos estudados. Muitas outras associações têm sido descritas, no entanto, sem comprovação clínica bem definida.

Assim, relacionando os dados encontrados, verificase que a incidência de colelitíase em nosso meio é compatível com os valores relatados pela literatura. No que se refere a outras patologias hepáticas, pôde-se estabelecer uma relação etiológica bem definida com a colelitíase, em especial, para os casos de neoplasias, infarto hepático e atrofia parda do fígado.

\begin{abstract}
Background: the aim of the present study is to evaluate the incidence of cholelithiasis on necropsies performed at the Unicamp Clinical Hospital and relate them to other associated diseases. Method: The authors analyzed the incidence of cholelithiasis on 2355 autopsies performed at the Pathological Anatomy Department of UNICAMP, between 1975 and 1998, among patients older than 10 years old. Chi-square and odds ratio tests were used to analyze correlation with other diseases. Results: There were 243 (10,3\%) cases of cholelithiasis, in 110 men $(7,9 \%)$ and $133(13,6 \%)$ women $(p=0,00001)$, a proportion of 1:17. This incidence increased with age ( $p<0,000000001)$, and differed significantly between ethnical groups being, $195(11,1 \%)$ among whites and 48 $(7,8 \%)$ among non whites $(p=0,02)$. Regarding other hepatic diseases, estheatosis was present in 33 (13,5\%), malignant neoplasms in 31 (12,7\%); cirrhosis in 30 (12,3\%), virus hepatitis and cronical liver congestion in 16 each (6,5\%). Malignant neoplasm, liver infarction, and hepatic dystrophic disease showed a significantly statistical association with the incidence of cholelithiasis. Only seven $(0,29 \%)$ cases of gallbladder cancer were detected. Conclusions: Our results show a progressive raise on the incidence of cholelitiasis with aging, being more common on the population over 80 years old, and with a white ethnical background.
\end{abstract}

Key Words: Cholelithiasis; Gallbladder; Necropsies. 


\section{REFERÊNCIAS}

1. Barker DJP, Gardner MJ, Power C, et al. Prevalence of gallstones at necropsy in nine British towns: a collaborative study. British Medical J, 1979, 2:1389-92.

2. Brett M, Barker DJP. The world distribution of gallstone. Int J Epidemiol, 1976, 5:335-41.

3. Burkitt DP, Tunstall M. Gallstones geographical and chronological features. J Trop Med Hyg, 1975, 78:140-4

4. Lagman MIS. The epidemiology of chronic digestive disease: gallstones. London - Edward Arnold, 1979.

5. Rocha A. Litíase da vesícula biliar em material de autópsia no Triângulo Mineiro. Rev Assoc Med Brasil, 1977, 23:196-8.

6. Nakaie M, Bevilacqua RG, Birolini D. Incidência de colelitíase em autópsias no município de São Paulo. Rev Paul Med, 1982, 2:11-5.

7. Jallut A, Hostetter R, Gardiol D. La lithiase biliaire dans un Collectif d'Autopsies. Schweiz Rundschau Med Prax, 1991, 80:615-9.

8. Jensen $\mathrm{KH}$, Jorgensen $\mathrm{T}$. Incidence of gallstones in a Danish population. Gastroenterology, 1991, 100:790-4.

9. Newman HF, Northup JD. The autopsy incidence of gallstones. Int Abst Surg, 1959, 109:1-13.

10. Coelho VCU, Bonelho R, Pitoki SAM, et al. Prevalence of gallstones in Brazilian population. Int Surg, 1999, $84: 25-8$.

11. Barbara L, Sama C, Labate AMM, et al. A population study on the prevalence of gallstone disease: The Sirmione Study. Hepatology, 1987, 7:913-7.

12. Lieber MD, Marshal M. The incidence of gallstones and their correlation with other disease. Ann Surg, 1952, 135:394-405.

13. Genzini T, Miranda MP, Silva A, et al. Cholelithiasis in cirrhotic patients. (analysis of cholelithiasis among patients with liver cirrhosis in São Paulo, Brazil). Arq Gastroenterol, 1996, 33:52-9.

14. Burnett W. The epidemiology of gallstones. Tijdschr Gastroenterol, 1971, 14:78-9.
15. Sternby NH. Atherosclerosis in a defined population. An autopsy study in Malmo, Sweden. Acta Pathol Microbiol Scand, 1968, 194:136-42.

16. Bergman F, Van Der Linden W, Söederströn J. The connection between myocardial infarction and gallstones in a autopsy series. Acta Path Microbiol Scand, 1968, 73:559-64.

17. Tandon BN. Patients in India with cirrhosis do not have increased prevalence gallstones. Am J Gastroenterol, 1989, 83:663.

18. Iber FL, Caruso G, Popepalle C, et al. Increasing prevalence of gallstones in male veterans with alcoholic cirrhosis. Am J Gastroenterol, 1990, 85:1593-96.

19. Acalovschi M, Badea R, Pascu M. Incidence of gallstones in liver cirrhosis. Am J Gastroenterol, 1991, 86:117981.

20. Del Omo JA, Garcia F, Serra MA, et al. Prevalence and incidence of gallstones in liver cirrhosis. Scand $\mathbf{J}$ Gastroenterol, 1997, 32:1061-65.

21. Fonari F, Imberti D, Squillante MM, et al. Incidence of gallstones in a population of patients with cirrhosis. Journal of Hepatology, 1994, 20:797-801.

Endereço para correspondência:

Dr. Mário Mantovani

Rua José de Campos Salles, 650

13095-300 - Campinas-SP

\section{COLÉGIO BRASILEIRO DE CIRURGIÕES}

www.cbc.org.br

e-mail:cirurgioes@openlink.com.br 